\title{
New insights into rhythmic brain activity from TMS-EEG studies
}

\author{
Gregor Thut ${ }^{1}$ and Carlo Miniussi ${ }^{2,3}$ \\ ${ }^{1}$ Centre for Cognitive Neuroimaging, Department of Psychology, 58 Hillhead Street, Glasgow G12 80B, UK \\ ${ }^{2}$ Department of Biomedical Sciences and Biotechnology, University of Brescia, Viale Europa 11, 25123, Brescia, Italy \\ ${ }^{3}$ Cognitive Neuroscience Section, IRCCS San Giovanni di Dio Fatebenefratelli, Via Pilastroni 4, 25125, Brescia, Italy
}

\begin{abstract}
There is renewed interest in the functional role of oscillatory brain activity in specific frequency bands, investigated in humans through electroencephalography (EEG) and magnetoencephalography (MEG) recordings. In parallel, there is a growing body of research on noninvasive direct stimulation of the human brain via repetitive (rhythmic) transcranial magnetic stimulation (TMS), and on those frequencies that have the strongest behavioural impact. There is, therefore, great potential in combining these two lines of research to foster knowledge on brain rhythms, in addition to potential therapeutic applications of rhythmic brain stimulation. Here, we review findings from this rapidly evolving field linking intrinsic brain oscillations to distinct sensory, motor and cognitive operations. The findings emphasize that brain rhythms are causally implicated in cognitive functions.
\end{abstract}

\section{Basic organizing principles of brain rhythms: a brief introduction}

Rhythmic activity is a fundamental property of neural elements and is organized in complex patterns depending on the state of the brain (e.g. sleep or awake) and on the task that is currently being executed. It can be recorded non-invasively from the scalp of human participants by electroencephalography (EEG) or magnetoencephalography (MEG) and many of the oscillatory components of EEG and MEG signals have been attributed a role in a variety of brain operations, including aspects of perception, cognition and action. One of the organizing principles of rhythmic activity is synchronization of oscillations across neuronal elements. This can occur locally between neurons within an area or over longer distances between areas within a wider network (e.g. see Refs [1-3]). Another characteristic of rhythmic brain activity is its frequency (for frequency bands used to classify brain oscillations see Glossary). Brain oscillations range from $\sim 0.05$ to $600 \mathrm{~Hz}$ [4], with slow wave activity being associated with sleep and faster oscillations with the awake state. Oscillatory activity in specific frequency bands has, therefore, been related to distinct functions. In terms of perception, for instance, $\gamma$-oscillations $(30-100 \mathrm{~Hz})$ have been linked to perceptual grouping and maintenance in visual memory (e.g. see Ref. [5]). In contrast, the slower $\theta(4-8 \mathrm{~Hz})$ and $\alpha$ oscillations $(8-14 \mathrm{~Hz})$ have been related to interregional,

Corresponding author: Thut, G. (g.thut@psy.gla.ac.uk). long-distance interaction for control of lower-level by higher-order areas [6-8] or for unification of cognitive operations through phase-coupling with other frequency bands $[9,10]$, besides more local processes (see later).

Despite recent advances, the functional meaning of these frequencies and their interaction remains a field of intense research. Here, we illustrate how the combination of EEG with rhythmic brain stimulation (i.e. transcranial magnetic stimulation [TMS] or transcranial alternating current stimulation [tACS]) (Box 1), which is now feasible through recent technical developments (e.g. see Refs $[11,12])$, holds promise for advancing our understanding of the brain rhythms underlying cognition.

\section{Analogies between EEG-, MEG- and TMS-research on rhythmic brain activity: added value of combining these techniques}

There is renewed interest in ongoing oscillatory brain activity in the absence of any stimulus input or motor output as an index of the internal state of the brain, and linked to it, the extent to which features of this ongoing activity have predictive power for subsequent sensory experience or cognitive processes (e.g. see Ref. [13]). This interest in how the internal state of the brain shapes forthcoming perception and cognition transpires many fields in neuroscience (for a recent review see Ref. [14]). Within this framework, accumulating EEG and MEG evidence has allowed for investigations of the relationship between the spectral content of oscillatory activity immediately before the appearance of a visual stimulus and its perceptual fate both in man [8,15-17] and monkey [18]. This approach has attracted much interest as it provides a means to address the question of the extent to which fluctuations in pre-stimulus (baseline) activity represent

\section{Glossary}

Electroencephalography (EEG) and magnetoencephalography (MEG): noninvasive electrophysiological recording techniques that sample ongoing electrical brain activity, which oscillates at various frequencies, through electrodes or sensors placed over the whole scalp.

Frequency bands used to classify brain oscillations: $\delta$ : delta $(0.5-4 \mathrm{~Hz}), \theta$ : theta $(4-8 \mathrm{~Hz}), \alpha$ : alpha $(8-14 \mathrm{~Hz}), \beta$ : beta $(14-30 \mathrm{~Hz}), \gamma$ : gamma $(30-100 \mathrm{~Hz})$, fast $(100$ $200 \mathrm{~Hz})$ and ultra fast $(200-600 \mathrm{~Hz})$.

Transcranial magnetic stimulation (TMS) or transcranial alternating current stimulation (tACS): techniques that allow for non-invasive rhythmic stimulation of the human brain at specific frequencies, such as those that can be recorded via EEG and MEG. 


\section{Box 1. Rhythmic brain stimulation}

Transcranial magnetic stimulation (TMS), illustrated in Figure la, is an instrument that can be used to investigate the brain-behaviour relationship and explore the excitability of different regions of the brain.

The technique involves delivering a brief, single, high-intensity magnetic pulse to the head through a coil. This induces electrical currents in a focal area underneath the coil, which interacts with ongoing activity in the neural tissue. These brief currents can transiently influence behaviour by producing excitation or inhibition of the stimulated cortical area [74]. Technical development has made available devices that are capable of delivering several pulses in rapid sequence up to $100 \mathrm{~Hz}$ (repetitive TMS [rTMS]). rTMS offers the opportunity to interact even more effectively with cortical activity. It has been shown that by using rTMS it is possible to transiently modulate neural excitability, with the net effect depending on the stimulation frequency. Usually, low frequency $(\leq 1 \mathrm{~Hz})$ results in inhibition, whereas high frequency stimulation can result in excitatory changes in the stimulated area [19].

A recently introduced technique called transcranial alternating current stimulation (tACS) (Figure lb) involves applying weak electrical currents to the head. The currents generate an electromagnetic field that modulates neuronal activity. Electrical stimulation is delivered with a battery-driven stimulator by means of a large electrode located on the area of interest and a reference electrode that is placed over a neutral area. Current research indicates that tACS entrains specific EEG frequency bands and induces phenomena specifically connected to the functions of the stimulated region and its oscillatory activities (i.e. tACS can interact with ongoing rhythmic brain activity in a frequency specific fashion) [24].

Both these types of rhythmic brain stimulation could provide the basis to interact with or induce local oscillatory activity, serving as a tool to investigate the role of ongoing brain rhythms at different frequencies.

(a)

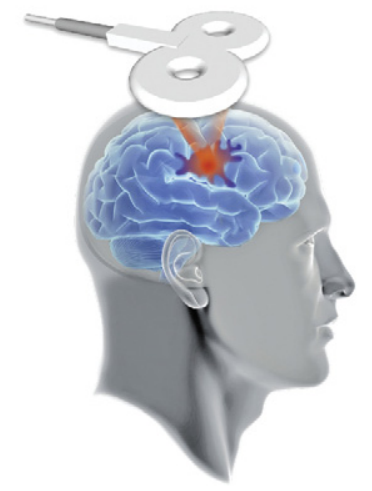

(b)

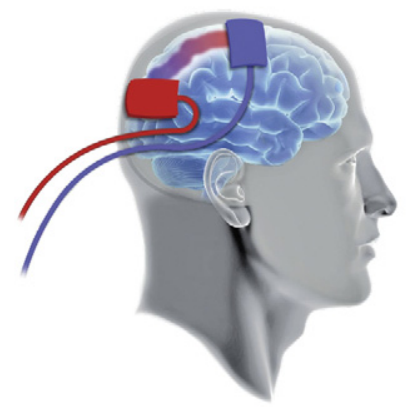

$\overline{\text { TRENDS in Cognitive Sciences }}$

Figure I. (a) TMS. When researchers operate a TMS coil near a participant's scalp, a powerful $(\sim 2 \mathrm{~T})$ and rapidly $(\sim 300 \mu \mathrm{s})$ changing magnetic field passes painlessly through skin and bone. Because the strength of the magnetic field falls off very rapidly with distance from the TMS coil, it can penetrate only a few centimetres and this means that only superficial areas of the brain are most effectively stimulated. The induced electric field causes electric current in nearby neurons, thus stimulating targeted regions of the cortex. (b) tACS. This method relies on application of alternating currents through an electrode. Electrical currents are applied constantly at low intensities (1 $\mathrm{mA}$ ) over a period of time in the order of seconds, to achieve changes in cortical activity. The waveform of the stimulation is sinusoidal and different frequencies can be used during stimulation (up to $250 \mathrm{~Hz}$ ).

noise or carry functional meaning. By linking local, prestimulus spectral content to performance of specific aspects of a task, the approach can also provide new information on potential functional specificity of brain rhythms. The find- ing of such links would be suggestive of rhythmic activity conditioning perception and cognition, as compared to merely being a by-product of the underlying mechanisms (e.g. see Ref. [4]), that is, of pre-stimulus brain activity causally shaping and not only correlating with upcoming perception.

Similar to the aforementioned EEG and MEG studies, there is a long tradition of research on rhythmic brain stimulation via TMS [19,20], and more recently tACS [21], to modulate ongoing neuronal activity locally and thus to condition behaviour. Although stimulation with frequencies such as $5 \mathrm{~Hz}$ (within $\theta$-band), $10 \mathrm{~Hz}$ (within $\alpha$-band) or $20 \mathrm{~Hz}$ (within $\beta$-band) seems to have a similar (temporary excitatory) outcome, these effects have mostly been investigated in the context of clinical use of repeated trains of rhythmic TMS (e.g. see Ref. [19]). Only a few studies have explored the effects of potential frequency 'entrainment' during or shortly after a single train of rhythmic stimulation, showing frequency-specific effects on perceptual or cognitive task performance [22-24].

Combining research on brain rhythms by EEG, MEG and rhythmic brain stimulation is therefore promising in several ways. On the one hand, the combination can provide a more in-depth picture on how brain oscillations relate to cognitive activities. In particular, it allows not only for an anatomofunctional mapping of oscillatory brain activity but also can provide new information on the nature of the link between brain rhythms and cognition (causal vs. correlative). On the other hand, the combination has the potential to give way to new ideas on how to interact with neural elements and networks in their own way of communication (i.e. through inducing oscillations) with the aim of manipulating functions. Here, we review new findings on these aspects that start to emerge from combined TMS-EEG studies. Because many of these studies have yielded results on the $\alpha$-frequency band [22,25-31], which is the most prominent rhythm in the awake state in terms of amplitude (strongest over posterior and central scalp recording sites overlying occipito-parietal and sensorimotor areas), there is an emphasis on this rhythmic activity throughout this review.

\section{Do specific frequencies reflect specific functions? $\alpha$ - oscillations and the regulation of cortical excitability versus inhibition}

Recent EEG and MEG research has identified posterior $\alpha$ oscillations, recorded before a visual event, as a predictor of the perceptual fate of the stimulus [8,15-17]. More specifically, the amplitude of $\alpha$-oscillations over occipito-parietal sites is inversely related to perception of the forthcoming visual event [8,15-17] (Figure 1a) with enhancement of $\alpha$ power being observed under conditions requiring suppression of task-irrelevant visual information [32-35]. Based on these findings, posterior $\alpha$-band changes have been proposed to have a role in perceptually relevant tuning of occipito-parietal areas in the anticipation of visual events (e.g. see Refs [16,17]), serving the regulation of the incoming flow of information along the dorsal stream. This is likely to go along with changes in receptivity within these areas [16], possibly by modulating signal-gain control of the forthcoming visual input [17]. 


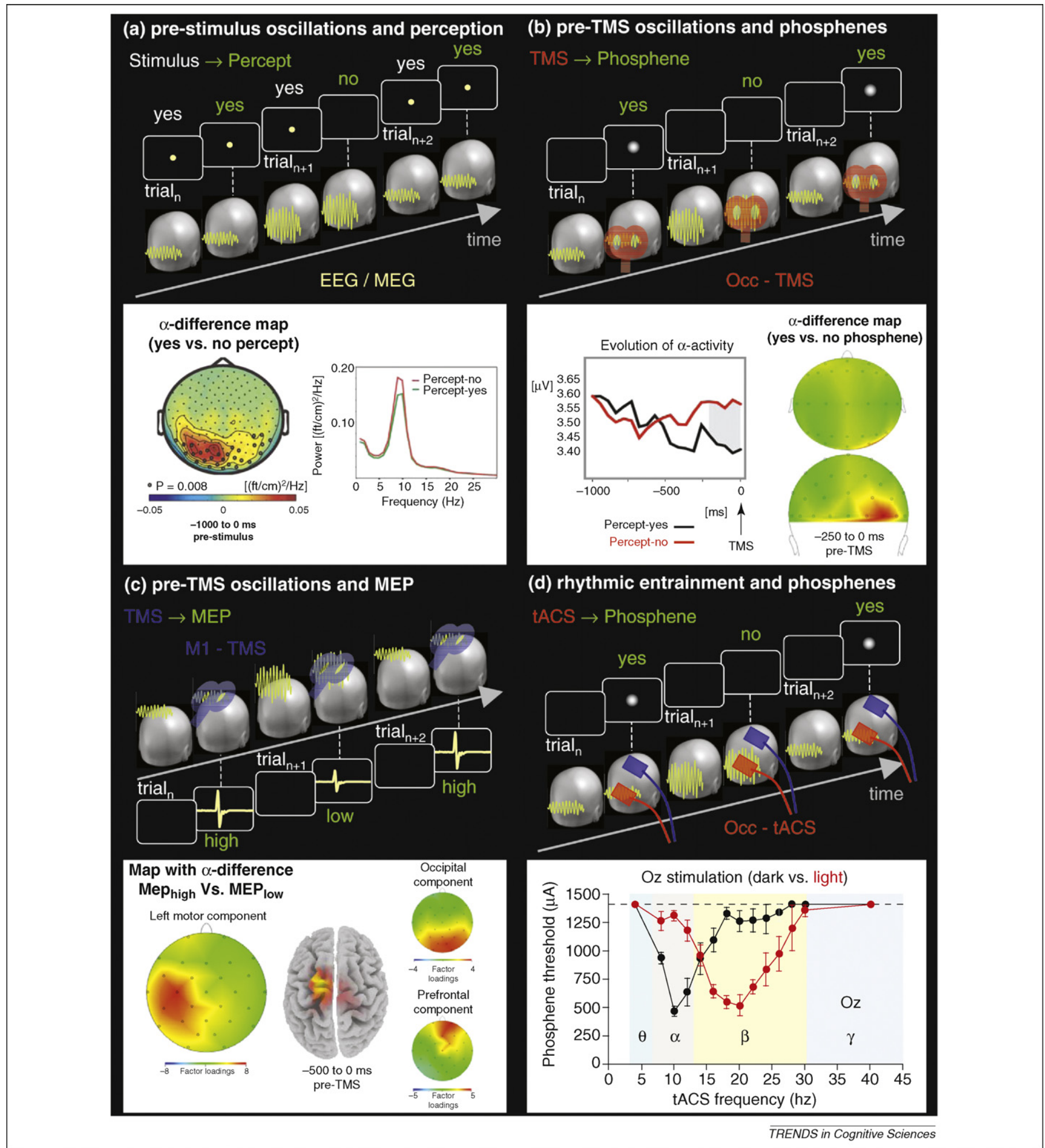

Figure 1. Recent findings of a (a-c) correlative and (d) causal link between oscillatory $\alpha$-band activity and behaviour, illustrating the interest for combining transcranial brain stimulation and EEG-research to advance our understanding of brain rhythms. (a) Magnetoencephalography: participants were presented with near-threshold stimuli and asked to report their visual percepts, while oscillatory brain activity was recorded. Specific oscillatory signatures before stimulus onset were related to whether the stimulus was perceived or not (schematic representation). Perceptually relevant signatures were confined to the occipito-parietal recording sites (see map of spectral power difference) and to the $\alpha$-frequency band (see spectral power curves). Low pre-stimulus power was predictive of hits, whereas high pre-stimulus power was predictive of misses. Adapted, with permission, from Ref. [17]. (b) Transcranial stimulation and Electroencephalography I: probing the excitability of the visual cortex via its direct stimulation through occipital single-pulse TMS (evoking sensations of lights, called phosphenes, without retinal input), while oscillatory activity was simultaneously recorded (schematic representation). Low $\alpha$ activity at the time of TMS was indicative of a highly excitable visual cortex (in terms of the likelihood of inducing phosphenes, black line, Phosphene-yes trials), whereas high- $\alpha$ activity was indicative of a less excitable cortex (red line, Phosphene-no trials). The perceptually relevant $\alpha$ changes were localized to occipital electrodes under the TMS coil opposite to the perceived phosphenes (see map, right-lateralized TMS). Adapted, with permission, from Ref. [25]. (c) Transcranial stimulation and Electroencephalography II: analogous to (b), but probing whether variability in motor cortex excitability (tested through the size of MEPs evoked by single-pulse TMS over the motor cortex) is linked to spontaneous $\alpha$-band fluctuations (schematic representation). Rolandic $\alpha$-band changes were inversely linked to motor cortex excitability (low $\alpha$ predicting high excitability and high $\alpha$ low excitability) (see left map), whereas other $\alpha$ components were unrelated (right maps). Adapted, with permission, from Ref. [27]. (d) Transcranial rhythmic stimulation: direct rhythmic stimulation of the visual cortex (via tACS) evoked phosphenes only when the 
Recently, TMS-EEG studies have provided further support for this notion. Direct evidence for a link between local $\alpha$-oscillations and local neuronal excitability has been obtained by directly stimulating the human occipital cortex via single-pulse TMS, while simultaneously recording EEG. Over posterior recording sites, spontaneous fluctuations of oscillatory activity have been shown to co-vary with the likelihood of occipital TMS to induce sensations of light (phosphenes) [25] (Figure 1b), thought to result from early visual cortex (V1/V2) stimulation [36]. An inverse relationship between pre-TMS $\alpha$-power and neuronal excitability in visual areas was observed. High $\alpha$-amplitude over posterior sites indexed low likelihood of inducing phosphenes, and low $\alpha$-amplitude a high likelihood, both over trials [25] (Figure 1b) and across participants [26].

In parallel, TMS-EEG studies have assessed the relationship between EEG fluctuations and the excitability of the motor cortex [27-29] (the latter derived from the size of peripheral motor-evoked potentials [MEPs] induced by TMS over primary motor cortex [M1]). In analogy to the aforementioned finding on $\alpha$ and TMS-probed visual cortex excitability, Sauseng et al. [27] revealed an inverse link between spontaneous fluctuations of $\alpha$-power recorded close to the central (rolandic) sulcus and the peripheral MEP-magnitude (Figure 1c) (corroborating Ref. [28], but see also Refs $[37,38]$ for contradictory evidence). Instead of probing for a link between $\alpha$ and cortical excitability in spontaneous fluctuations, Brignani et al. [29] studied the co-modulation of oscillatory brain activity and TMS-probed cortical excitability after administration of a TMS protocol (1Hz-stimulation over several minutes) that is known to have an inhibitory impact on the excitability of the targetarea (e.g. see Ref. [19]). Applied over left M1, this protocol resulted in local (left rolandic) $\alpha$-power enhancement, which was correlated with a simultaneous decrease in TMS-probed M1-excitability (i.e. with the inhibitory impact of the 1Hz-protocol [29]).

These TMS-EEG studies thus extend research on the $\alpha$ rhythm by indicating that local $\alpha$-amplitude carries information about the momentary (excitability) state of neurons within the dorsal processing stream. The findings show that up- and down-regulation of $\alpha$, possibly involving top-down control, is likely to condition the cortex for forthcoming perception or action. This has added to a mounting body of information on the role of $\alpha$-oscillations in attentional selection. Posterior $\alpha$-power in retinotopically organized (visual) areas [34,39] of the occipital and parietal lobes [40] is modulated by deployment of visual attention to specific positions in space, with $\alpha$-power being downregulated in the hemisphere opposite to the attended location $[7,16,35,40,41]$ and up-regulated opposite to the unattended portion of space [33-35]. Over posterior areas, the $\alpha$-variations thus seem to regulate the flow of incoming information $[17,25,26,32,42]$, whereas over rolandic areas, modulation of oscillatory $\alpha$-band activity might in addition be instrumental for the control of transforming perceptions or acquired sensorimotor memories into action $[43,44]$.

\section{Topography of brain-rhythms: are $\alpha$-oscillations intrinsic to specific brain regions?}

If specific frequencies have specific functions, they should show some degree of topographic specificity. Over the scalp, the cerebral $\alpha$-rhythm is predominant over posterior and rolandic sites (over rolandic sites alongside $\beta$-oscillations). It is modulated by sensory input or motor output and therefore thought to reflect the spontaneous rhythm of sensory and sensory-motor areas. In line with this view, it has been found to originate in calcarine, occipito-parietal and somatosensory cortex $[45,46]$, generated through complex cortico-cortical and thalamo-cortical interactions [47], although other cortical sources including auditory cortex, areas of the ventral visual stream [45] and mechanisms of generation exist (e.g. see Ref. [48]). If topographically specific, the $\alpha$-rhythm should be differentially affected by direct cortical stimulation via TMS, depending on stimulation sites. The following TMS-EEG findings support this view.

Applying single-pulse TMS to the motor cortex triggers transient neuronal oscillations in the $\alpha$ - and/or $\beta$-frequency bands $[30,49,50]$, with mixed results as to which band is more reactive ( $\alpha$-band in Ref. [30]; $\beta$-band in Ref. [50]). Importantly, the observed synchronization of neuronal activity was found to show topographic specificity because it was less strong in response to stimulation of dorsal premotor cortex than M1-stimulation [50]. It has been proposed that the induced oscillations emerge because of a reset of the stimulated area's spontaneous rhythm, thereby offering a unique approach to study the generation of oscillatory activity in the human brain $[30,49,50]$.

Additional information as to the topographic specificity of rhythmic $\alpha$ (and $\beta$ ) generators can be gained from post-hoc analysis of those TMS-EEG studies that investigated the EEG-changes in the minutes that follow administration of repeated TMS pulses (rTMS). Thus far, such rTMS-induced aftereffects have been quantified in a total of 14 studies (Figure 2). A 'meta'-analysis points towards a consistent topographical pattern of frequency changes. Across the 10 studies that stimulated either M1 [29,51-54], V1/V2 [55] or dorsolateral prefrontal cortex (DLPFC) [56-59], there is a bias for $\alpha$ - and $\beta$-oscillations to be affected more often after sensory or motor cortex stimulation than after DLPFC-stimulation $(n=6 / 6$ studies versus $n=1 / 4$ studies). Aftereffects in other frequency bands $(\delta, \theta)$ are associated more often with DLPFC than sensory or motor cortex stimulation $(n=3 /$ 4 studies versus $\mathrm{n}=0 / 6$ studies). This differential distribution of aftereffects as a function of stimulation site and oscillations reaches statistical significance (according to a chi-square test: $p<0.05$ ), although it might partially be explained by a focus on the analysis of $\alpha$ and/or $\beta$-activity in M1 studies (but see Refs [51] and [58] for 'doubledissociations' of $\alpha$ - versus $\theta$-aftereffects after M1- versus DLPFC-stimulation). Note also that although stimulation with rTMS was focal, the observed effects were widespread, including many recording sites (Figure 2), 


\begin{tabular}{|c|c|c|c|c|c|c|}
\hline \multirow{7}{*}{ M1 } & Study & $\begin{array}{l}\text { Healthy } \\
\text { Subjects }\end{array}$ & $\begin{array}{l}\text { TMS- } \\
\text { protocol }\end{array}$ & $\begin{array}{l}\text { Outcome- } \\
\text { measures }\end{array}$ & $\begin{array}{l}\text { MEG/EEG- } \\
\text { aftereffect }\end{array}$ & $\begin{array}{l}\text { MEG/EEG-sites } \\
\text { of aftereffect }\end{array}$ \\
\hline & Strens et al.[51] & $N=15$ & $\begin{array}{l}1 \mathrm{~Hz} / 1 \times 25 \mathrm{~min} \\
\text { M1 }\end{array}$ & $\begin{array}{l}\text { spectral power } \\
\text { coherence } \\
\text { (rest and task) }\end{array}$ & $\begin{array}{l}\text { focal coherence increase } \\
\text { (alpha-band) }\end{array}$ & $\begin{array}{l}\text { frontal- } \\
\text { central- } \\
\text { parietal }\end{array}$ \\
\hline & Oliviero et al.[52] & $N=16$ & $\begin{array}{l}5 \mathrm{~Hz} / 1 \times 10 \text { s train } \\
\text { M1 }\end{array}$ & $\begin{array}{l}\text { spectral power } \\
\text { coherence } \\
\text { (rest and task) }\end{array}$ & $\begin{array}{l}\text { focal coherence decrease } \\
\text { (alpha-band) }\end{array}$ & $\begin{array}{l}\text { frontal- } \\
\text { central- } \\
\text { parietal }\end{array}$ \\
\hline & Tamura et al..[53] & $N=12$ & $\begin{array}{l}1 \mathrm{~Hz} / 1 \times 10 \mathrm{~min} \\
\text { M1 }\end{array}$ & $\begin{array}{l}\text { movement-related } \\
\text { oscillations (task) }\end{array}$ & $\begin{array}{l}\text { decrease in synchronization } \\
\text { (beta-band) }\end{array}$ & central \\
\hline & Fuggetta et al.[54] & $N=11$ & $\begin{array}{l}5 \mathrm{~Hz} / 20 \times 4 \mathrm{~s} \text { train } \\
\mathrm{M} 1\end{array}$ & $\begin{array}{l}\text { EEG power and } \\
\text { coherence (rest) }\end{array}$ & $\begin{array}{l}\text { short-lived focal incr/decr } \\
\text { in power/coherence } \\
\text { (alpha \& beta) }\end{array}$ & $\begin{array}{l}\text { frontal- } \\
\text { central- } \\
\text { parietal }\end{array}$ \\
\hline & Brignani et al.[29] & $N=6$ & $\begin{array}{l}1 \mathrm{~Hz} / 1 \times 10 \mathrm{~min} \\
\mathrm{M} 1\end{array}$ & $\begin{array}{l}\text { EEG power } \\
\text { (rest) }\end{array}$ & $\begin{array}{l}\text { focal, cumulative increase in } \\
\text { power (alpha-band) }\end{array}$ & central \\
\hline & Thut et al.[55] & $N=6$ & $\begin{array}{l}1 \mathrm{~Hz} / 10 \mathrm{~min} \\
\mathbf{V} 1 / \mathrm{V} 2\end{array}$ & $\begin{array}{l}\text { visual induced } \\
\text { desynchronization } \\
\text { (task) }\end{array}$ & $\begin{array}{l}\text { less desynchronization } \\
\text { (alpha-band) }\end{array}$ & $\begin{array}{l}\text { parieto- } \\
\text { occipital }\end{array}$ \\
\hline \multirow[t]{4}{*}{ DLPFC } & $\begin{array}{l}\text { Jing and } \\
\text { Takigawa[56] }\end{array}$ & $N=19$ & $\begin{array}{l}10 \mathrm{~Hz} / 2 \times 3 \mathrm{~s} \text { train } \\
\text { DLPFC }\end{array}$ & $\begin{array}{l}\text { coherence } \\
\text { (rest) }\end{array}$ & $\begin{array}{l}\text { focal directed coherence } \\
\text { increase (alpha-band) }\end{array}$ & $\begin{array}{l}\text { frontal- } \\
\text { parietal }\end{array}$ \\
\hline & Okamura et al..[57]. & $\mathrm{N}=32$ & $\begin{array}{l}10 \mathrm{~Hz} / 2 \times 3 \text { s train } \\
\text { DLPFC }\end{array}$ & $\begin{array}{l}\text { spectral power } \\
\text { (rest) }\end{array}$ & $\begin{array}{l}\text { change in peak frequency and } \\
\text { power (many frequencies) }\end{array}$ & $\begin{array}{l}\text { many electrode } \\
\text { sites }\end{array}$ \\
\hline & Schutter et al.[58] & $N=12$ & $\begin{array}{l}1 \mathrm{~Hz} / 1 \times 20 \mathrm{~min} \\
\text { DLPFC }\end{array}$ & $\begin{array}{l}\text { spectral power } \\
\text { (rest) }\end{array}$ & $\begin{array}{l}\text { increase in power } \\
\text { (theta-band) }\end{array}$ & prefrontal \\
\hline & Griskova et al.[59] & $N=18$ & $\begin{array}{l}10 \mathrm{~Hz} / 100 \times 2 \mathrm{~s} \text { train } \\
\text { DLPFC }\end{array}$ & $\begin{array}{l}\text { spectral power } \\
\text { (rest) }\end{array}$ & increase in power (delta-band) & $\begin{array}{l}\text { frontal } \\
\text { central } \\
\text { parietal }\end{array}$ \\
\hline \multirow{4}{*}{ Cerebellum } & Klimesch et al.[22] & $\mathrm{N}=16$ & $\begin{array}{l}\mathrm{IAF}-20 \mathrm{~Hz} \\
1.2-4.8 \mathrm{~s} \text { trains } \\
\text { parietal and frontal }\end{array}$ & $\begin{array}{l}\text { event-related } \\
\text { desynchronization }\end{array}$ & $\begin{array}{l}\text { increase of } \\
\text { desynchronization (alpha) }\end{array}$ & $\begin{array}{l}\text { parieto- } \\
\text { occipital }\end{array}$ \\
\hline & Chen et al.[60] & $N=8$ & $\begin{array}{l}0.9 \mathrm{~Hz} / 1 \times 15 \mathrm{~min} \\
\mathrm{PM}\end{array}$ & $\begin{array}{l}\text { movement-related } \\
\text { power/coherence } \\
\text { (task) }\end{array}$ & $\begin{array}{l}\text { less task-related decrease } \\
\text { (alpha \& beta) } \\
\text { coherence increase (alpha) }\end{array}$ & central \\
\hline & Schutter et al.[63] & $N=5$ & $\begin{array}{l}25 \mathrm{~Hz} / 80 \times 10 \text { s train } \\
\text { Cerebellum }\end{array}$ & $\begin{array}{l}\text { spectral power } \\
\text { (rest) }\end{array}$ & $\begin{array}{l}\text { change in asymmetry } \\
\text { (gamma-band) }\end{array}$ & prefrontal \\
\hline & Pastor et al.[64] & $N=6$ & $\begin{array}{l}1 \mathrm{~Hz} / 1 \times 10 \mathrm{~min} \\
\text { Cerebellum }\end{array}$ & $\begin{array}{l}\text { steady-state } \\
\text { Potentials }\end{array}$ & $\begin{array}{l}\text { change in } \\
\text { oscillations (gamma-band) }\end{array}$ & prefrontal \\
\hline
\end{tabular}

Figure 2. Aftereffects on brain oscillations following the administration of repeated TMS pulses. Stimulation of early visual (V1/V2) or primary motor cortex (M1) most frequently affected local oscillatory activity in the $\alpha$ - and $\beta$-bands $[29,51-55]$, whereas stimulation of the dorsolateral prefrontal cortex (DLPFC) often interfered with oscillations in other bands [56-59]. Aftereffects were widespread including effects on parieto-occipital $\alpha$-oscillations after frontal and parietal stimulation [22], rolandic $\alpha$ and

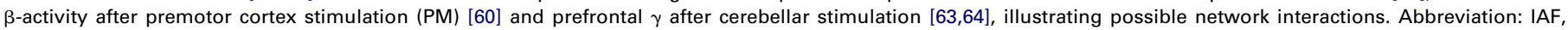
individual $\alpha$ frequency.

which can be explained by volume conduction because of the known coarse topographic resolution of EEG. Yet, this finding could also have resulted from the rTMS effect spreading from the target site to functionally connected areas.

Of note is a recent TMS-EEG study that used, for the first time, two TMS coils to apply synchronous rhythmic stimulation at $\alpha$-frequency over two sites [31]. The study revealed induced interregional coherence in the $\alpha$-frequency range between the stimulated hand motor and visual cortices, as compared to unifocal stimulation [31].

In conclusion, stimulating the cerebral cortex via TMS and studying what is triggered in terms of changes in brain oscillations can further advance our understanding of brain rhythms and their generation. In terms of $\alpha$-activity, TMS-EEG findings so far indicate that $\alpha$-rhythms can be entrained by TMS and reveal topographic specificity of this effect, although no cortical ' $\alpha$-map' is firmly established.

\section{Embedding in the cortical network: cortico-cortical interactions in the modulation of $\alpha$-activity}

If the $\alpha$-rhythm reflects oscillations of neuronal elements close to signal input- and output-stages as reviewed earlier, it should be adjustable through top-down control from higher-order areas involved in attention and movement control and thus depend on the integrity of these areas. Here, the combined TMS-EEG approach has provided new information by virtue of TMS over higher-order areas and the study of EEG-changes at remote, anatomically connected sites (akin to analogous TMS-studies on changes in evoked potentials [12]).

Capotosto et al. (NaturePrecedings, doi:10101/ npre.2008.1563.1) have studied the network interactions involved in posterior $\alpha$-changes during attention orienting in anticipation of a visual stimulus at pre-cued positions. TMS was applied over areas known to be involved in the control of visual spatial attention, namely frontal eye-field (FEF) and intraparietal sulcus (IPS). Both FEF- and IPS-stimulation led to a breakdown of 
the remote, asymmetric regulation of $\alpha$-activity contralateral to attended versus unattended space. This suggests that anticipatory $\alpha$-rhythms at posterior sites are controlled by signals from FEF and IPS and that disruption of this top-down control leads to suboptimal posterior oscillations.

Cortico-cortical and cerebello-cortical interactions in the generation of rhythmic activity have also been demonstrated by some of the TMS-EEG studies illustrated in Figure 2. In line with what has already been mentioned, changes in posterior $\alpha$-oscillations have been observed after either frontal or parietal TMS [22]. TMS to the lateral premotor cortex has been shown to affect rhythmic $\alpha$ activity over ipsilateral rolandic, and to a lesser extent, over contralateral rolandic sites [60]. Because fronto-parietal networks are regulating sensory or motor areas for attentional selection [61] and motor control [62] through their functional interconnectivity, these effects on $\alpha$ are likely to have originated downstream to stimulation.

It is also of interest to note that cerebellar stimulation so far has been observed to induce aftereffects on $\gamma$-oscillations at prefrontal sites, probably because of the functional connections from the cerebellum to the prefrontal cortex [63,64] (Figure 2).

\section{Rhythmic activity: epiphenomenal or causal manifestations of brain function?}

Do brain rhythms have a causal functional role or merely represent epiphenomenal manifestations of the processes underlying perception, cognition and action? Historically, the $\alpha$-rhythm was considered to reflect a cortical idling state, and its prominent suppression during visual and motor tasks [45] a valuable (but epiphenomenal) manifestation of cortical activation. Today, there is a growing body of evidence for $\alpha$-enhancement beyond baseline (idling) levels, speaking against this interpretation [65]. A more conclusive test is to use transcranial brain stimulation for rhythmic 'entrainment'. If, in terms of $\alpha$-oscillations, prestimulus activity is causally shaping perception, stimulation at $\alpha$-frequency for $\alpha$-'entrainment' (but not at other frequencies) should lead to perceptual consequences during or immediately after stimulation. There is, indeed, emerging evidence for such a causal link, coming from studies on the behavioural consequences of rhythmic stimulation within physiological parameters [22-24].

Rhythmic transcranial brain stimulation has been shown to selectively modulate perceptual processes as a function of stimulation frequency. In an innovative study on $\alpha$-'entrainment', Klimesch et al. [22] showed that frontal and parietal TMS at individual $\alpha$-frequency immediately before execution of a visual spatial task (mental rotation involving visual imagery and memory) affect task performance relative to stimulation in other frequency-bands. Using tACS, Kanai et al. [24] have shown that occipital stimulation most effectively induces phosphenes in darkness when applied at $\alpha$-frequency, whereas in the light $\beta$ stimulation was most effective (Figure 1d).

Rhythmic entrainment via transcranial brain stimulation has also proven to selectively affect declarative memory consolidation. During sleep, oscillatory activity at low frequencies $(<4 \mathrm{~Hz}$, slow wave activity) has been linked to overnight consolidation of memories because local changes in sleep slow-wave activity occur after day-time learning, and correlate positively with post-sleep performance improvement [66]. In line with a causal role of sleep slow-waves in memory consolidation, nocturnal TMS at $<1 \mathrm{~Hz}$ leads to enhanced slow wave activity in addition to deepening of sleep [67], and nocturnal tACS used with a DC-shift at slow wave frequency improves memory consolidation [23] (see Ref. [21] for more details regarding this stimulation protocol, nominated transcranial sinusoidal direct current stimulation [tSDCS] in Ref. [21]).

Thus, stimulation at physiologically meaningful rhythms has domain-specific effects on cognitive activities, which supports causal implication of cortical rhythms in cognitive function. Although TMS-EEG work so far has focused on a subset of brain rhythms and perceptual or cognitive processes, the various ways of using the TMS-EEG combination illustrated here are transferable to research on other oscillations and cognitive functions, as long as their generators are accessible via TMS. Many EEG- and MEG-studies have established links between oscillatory activity and higher cognitive activities other than those reviewed here. For instance, frontal midline $\theta$ activity has been identified as a correlate of working memory maintenance, of episodic memory encoding and retrieval (for review see Ref. [68]) and of emotional regulation (e.g. see Ref. [69]). Others have linked frontal $\alpha$-activity with insight problem solving [70] and specific electrographic signatures (e.g. long-range $\gamma$-synchronization) with access of information to consciousness [71]. A causal link between many of these rhythmic brain activities and their presumed functions remains to be elucidated.

\section{Box 2. Questions for future research}

- The bulk of the reviewed literature has focused on $\alpha$-power, its link to perception and action, and modulation through brain stimulation. Taking into account phase-information and post-stimulus periods in future research is likely to provide further crucial information on the function of $\alpha$-rhythmicity $[10,65]$.

- It is unknown how the reviewed $\alpha$-fluctuations relate to other oscillatory changes in sensory selection, such as those of higher $(\gamma)$ [18] or lower ( $\delta$ ) frequencies [75].

- It remains to be seen whether entraining specific oscillations such as posterior $\alpha$ by rhythmic brain stimulation could be helpful to promote neurorehabilitation. For example, because hemispatial neglect patients show asymmetric receptivity of otherwise intact sensory cortices [61], it is conceivable that these patients would profit from rhythmic entrainment over sensory areas to bias perception in desired direction.

- Although less prominent, $\alpha$-generators exist outside occipitoparietal and rolandic sites [45]. Their topographic origin (e.g. frontal $\alpha$ ) or their function (e.g. proposed implication in inhibitory control [65]) could be specifically tested by either $\alpha$-resetting through single-pulse TMS or by rhythmic entrainment protocols over these areas.

- Although rhythmic stimulation at frequencies $<30 \mathrm{~Hz}$ (slow wave, $\alpha$ and $\beta$ ) modulate performance [22-24], it is unknown whether the techniques that are available for non-invasive brain stimulation in humans can also entrain $\gamma$-oscillations in a physiologically meaningful pattern.

- Further empirical work is required to elucidate the role of brain oscillations in other aspects of higher cognitive functions, such as working memory, memory encoding and retrieval or consciousness $[2,3]$. 


\section{Rhythmic entrainment: the interest of biasing function in desired directions}

Could the previously mentioned findings lead to new implementations of rhythmic TMS as a tool in therapy and neurorehabilitation? Several investigations indeed indicate that interacting with cortical activity, by means of rhythmic transcranial stimulation, can positively influence cognitive performance of patients affected by disorders such as aphasia, unilateral neglect or dementia [72]. The modification of cortical activity for an adequate period of time through the use of rhythmic stimulation might adjust a post-lesionally established, but behaviourally maladaptive pattern of brain oscillation, and thus provide an opportunity for inducing a new balance within the affected functional network. The data reviewed here indicate that by pushing the network towards a new pattern of activation through restoring adequate synchronization, improvement of sensory and motor disorders or cognitive functions could be achieved. In line with this view, it has been demonstrated that neurofeedback training to enhance $\alpha$-oscillations generated in the auditory cortex (i.e. the tau-rhythm [45]) can help to reduce auditory sensations in patients suffering from tinnitus [73], possibly by locally enhancing inhibitory processes. Future brain stimulation (TMS or tACS) and EEG research should go hand-in-hand to further elucidate these and other outstanding questions (Box 2).

\section{Concluding remarks}

We have reviewed the evolving field of TMS-EEG research from which new insights into brain rhythms emerge. We have shown that the TMS-EEG combination is particularly promising and well suited to address several open questions on the generation and functional role of brain oscillations. This field has provided so far novel information mostly on the $\alpha$-rhythm, namely on its role in perceptually relevant tuning of occipito-parietal areas, on its origin of generation in sensory-motor regions and top-down modulation through higher-order areas. This is in line with the rapidly progressing understanding of this frequency's role in attentional selection. Overall, the findings emphasize that the frequency of neural discharges is not merely epiphenomenal. Extracting meaning from ongoing brain oscillations in the intact human brain and on the potential of rhythmic stimulation to selectively entrain frequencies in selected patients for restoring brain function will be a challenging field of research for years to come.

\section{Acknowledgements}

We thank Joachim Gross, Jan-Mathijs Schoffelen and three anonymous reviewers for comments, and Stefano Bonezzi for preparing Figure I in Box 1.

\section{References}

1 Womelsdorf, T. and Fries, P. (2007) The role of neuronal synchronization in selective attention. Curr. Opin. Neurobiol. 17, 154-160

2 Tallon-Baudry, C. (2004) Attention and awareness in synchrony. Trends Cogn. Sci. 8, 523-525

3 Ward, L.M. (2003) Synchronous neural oscillations and cognitive processes. Trends Cogn. Sci. 7, 553-559
4 Buzsáki, G. et al. (2004) Neuronal oscillations in cortical networks. Science 304, 1926-1929

5 Jensen, O. et al. (2007) Human gamma-frequency oscillations associated with attention and memory. Trends Neurosci. 30,317-324

6 von Stein, A. et al. (2000) Top-down processing mediated by interareal synchronization. Proc. Natl. Acad. Sci. U. S. A. 97, 14748-14753

7 Sauseng, P. et al. (2005) A shift of visual spatial attention is selectively associated with human EEG alpha activity. Eur. J. Neurosci. 22, 29172926

8 Hanslmayr, S. et al. (2007) Prestimulus oscillations predict visual perception performance between and within subjects. Neuroimage $37,1465-1473$

9 Jensen, O. and Colgin, L.L. (2007) Cross-frequency coupling between neuronal oscillations. Trends Cogn. Sci. 11, 267-269

10 Palva, S. and Palva, J.M. (2007) New vistas for alpha-frequency band oscillations. Trends Neurosci. 30, 150-158

11 Komssi, S. and Kähkönen, S. (2006) The novelty value of the combined use of electroencephalography and transcranial magnetic stimulation for neuroscience research. Brain Res. Rev. 52, 183-192

12 Taylor, P.C. et al. (2008) Combining TMS and EEG to study cognitive function and cortico-cortico interactions. Behav. Brain Res. 191, 141147

13 Engel, A.K. et al. (2001) Dynamic predictions: oscillations and synchrony in top-down processing. Nat. Rev. Neurosci. 2, 704-716

14 Silvanto, J. et al. (2008) State-dependency in brain stimulation studies of perception and cognition. Trends Cogn. Sci. 12, 447-454

15 Ergenoglu, T. et al. (2004) Alpha rhythm of the EEG modulates visual detection performance in humans. Brain Res. Cogn. Brain Res. 20, 376383

16 Thut, G. et al. (2006) Alpha-band electroencephalographic activity over occipital cortex indexes visuospatial attention bias and predicts visual target detection. J. Neurosci. 26, 9494-9502

17 van Dijk, H. et al. (2008) Prestimulus oscillatory activity in the alpha band predicts visual discrimination ability. J. Neurosci. 28 , 1816-1823

18 Fries, P. et al. (2001) Modulation of oscillatory neuronal synchronization by selective visual attention. Science $291,1560-1563$

19 Kobayashi, M. and Pascual-Leone, A. (2003) Transcranial magnetic stimulation in neurology. Lancet Neurol. 2, 145-156

20 Ridding, M.C. and Rothwell, J.C. (2007) Is there a future for therapeutic use of transcranial magnetic stimulation? Nat. Rev. Neurosci. 8, 559-567

21 Antal, A. et al. (2008) Comparatively weak after-effects of transcranial alternating current stimulation (tACS) on cortical excitability in humans. Brain Stimulation 1, 97-105

22 Klimesch, W. et al. (2003) Enhancing cognitive performance with repetitive transcranial magnetic stimulation at human individual alpha frequency. Eur. J. Neurosci. 17, 1129-1133

23 Marshall, L. et al. (2006) Boosting slow oscillations during sleep potentiates memory. Nature 444, 610-613

24 Kanai, R. et al. (2008) Frequency dependent electrical stimulation of visual cortex. Curr. Biol. 18, 1839-1843

25 Romei, V. et al. (2008) Spontaneous fluctuations in posterior alphaBand EEG activity reflect variability in excitability of human visual areas. Cereb. Cortex 18, 2010-2018

26 Romei, V. et al. (2008) Resting electroencephalogram alpha-power over posterior sites indexes baseline visual cortex excitability. Neuroreport 19, 203-208

27 Sauseng, P. et al. (2009) Spontaneous locally restricted EEG alpha activity determines cortical excitability in the motor cortex. Neuropsychologia 47, 284-288

28 Zarkowski, P. et al. (2006) EEG and the variance of motor evoked potential amplitude. Clin. EEG Neurosci. 37, 247-251

29 Brignani, D. et al. (2008) Modulation of cortical oscillatory activity during transcranial magnetic stimulation. Hum. Brain Mapp. 29, 603612

30 Fuggetta, G. et al. (2005) Modulation of cortical oscillatory activities induced by varying single-pulse transcranial magnetic stimulation intensity over the left primary motor area: a combined EEG and TMS study. Neuroimage 27, 896-908

31 Plewnia, C. et al. (2008) Enhancement of long-range EEG coherence by synchronous bifocal transcranial magnetic stimulation. Eur. J. Neurosci. 27, 1577-1583 
32 Jensen, O. et al. (2002) Oscillations in the alpha band $(9-12 \mathrm{~Hz})$ increase with memory load during retention in a short-term memory task. Cereb. Cortex 12, 877-882

33 Kelly, S.P. et al. (2006) Increases in alpha oscillatory power reflect an active retinotopic mechanism for distracter suppression during sustained visuospatial attention. J. Neurophysiol. 95, 3844-3851

34 Rihs, T.A. et al. (2007) Mechanisms of selective inhibition in visual spatial attention are indexed by alpha-band EEG synchronization. Eur. J. Neurosci. 25, 603-610

35 Rihs, T.A. et al. (2009) A bias for posterior alpha-band power suppression versus enhancement during shifting versus maintenance of spatial attention. Neuroimage 44, 190-199

36 Kammer, T. et al. (2005) Transcranial magnetic stimulation in the visual system. II. Characterization of induced phosphenes and scotomas. Exp. Brain Res. 160, 129-140

37 Lepage, J.F. et al. (2008) EEG and neuronavigated single-pulse TMS in the study of the observation/execution matching system: are both techniques measuring the same process? J. Neurosci. Methods 175, $17-24$

38 Mitchell, W.K. et al. (2007) Muscle responses to transcranial stimulation in man depend on background oscillatory activity. $J$. Physiol. 583, 567-579

39 Worden, M.S. et al. (2000) Anticipatory biasing of visuospatial attention indexed by retinotopically specific alpha-band electroencephalography increases over occipital cortex. J. Neurosci. 20, RC63

40 Siegel, M. et al. (2008) Neuronal synchronization along the dorsal visual pathway reflects the focus of spatial attention. Neuron 60, 709-719

41 Yamagishi, N. et al. (2008) Attentional changes in pre-stimulus oscillatory activity within early visual cortex are predictive of human visual performance. Brain Res. 1197, 115-122

42 Ploner, M. et al. (2006) Oscillatory activity reflects the excitability of the human somatosensory system. Neuroimage $32,1231-1236$

43 Pineda, J.A. (2005) The functional significance of mu rhythms: translating "seeing" and "hearing" into "doing". Brain Res. Brain Res. Rev. 50, 57-68

44 Hummel, F. et al. (2002) Inhibitory control of acquired motor programmes in the human brain. Brain 125, 404-420

45 Hari, R. et al. (1997) Magnetoencephalographic cortical rhythms. Int. J. Psychophysiol. 26, 51-62

46 Manshanden, I. et al. (2002) Source localization of MEG sleep spindles and the relation to sources of alpha band rhythms. Clin. Neurophysiol. 113, 1937-1947

47 Steriade, M. et al. (1990) Basic mechanisms of cerebral rhythmic activities. Electroencephalogr. Clin. Neurophysiol. 76, 481-508

48 Nunez, P.L. et al. (2001) Spatial-temporal structures of human alpha rhythms: theory, microcurrent sources, multiscale measurements, and global binding of local networks. Hum. Brain Mapp. 13, 125-164

49 Paus, T. et al. (2001) Synchronization of neuronal activity in the human primary motor cortex by transcranial magnetic stimulation: an EEG study. J. Neurophysiol. 86, 1983-1990

50 Van Der Werf, Y.D. and Paus, T. (2006) The neural response to transcranial magnetic stimulation of the human motor cortex. I. Intracortical and cortico-cortical contributions. Exp. Brain Res. 175, 231-245

51 Strens, L.H. et al. (2002) The effects of subthreshold $1 \mathrm{~Hz}$ repetitive TMS on cortico-cortical and interhemispheric coherence. Clin. Neurophysiol. 113, 1279-1285

52 Oliviero, A. et al. (2003) Persistent effects of high frequency repetitive TMS on the coupling between motor areas in the human. Exp. Brain Res. 149, 107-113
53 Tamura, Y. et al. (2005) Functional relationship between human rolandic oscillations and motor cortical excitability: an MEG study. Eur. J. Neurosci. 21, 2555-2562

54 Fuggetta, G. et al. (2008) Acute modulation of cortical oscillatory activities during short trains of high-frequency repetitive transcranial magnetic stimulation of the human motor cortex: a combined EEG and TMS study. Hum. Brain Mapp. 29, 1-13

55 Thut, G. et al. (2003) Differential effects of low-frequency rTMS at the occipital pole on visual-induced alpha desynchronization and visualevoked potentials. Neuroimage 18, 334-347

56 Jing, H. and Takigawa, M. (2000) Observation of EEG coherence after repetitive transcranial magnetic stimulation. Clin. Neurophysiol. 111, $1620-1631$

57 Okamura, H. et al. (2001) EEG modification induced by repetitive transcranial magnetic stimulation. J. Clin. Neurophysiol. 18, 318-325

58 Schutter, D.J. et al. (2001) Effects of slow rTMS at the right dorsolateral prefrontal cortex on EEG asymmetry and mood. Neuroreport 12, 445-447

59 Griskova, I. et al. (2007) The effects of $10 \mathrm{~Hz}$ repetitive transcranial magnetic stimulation on resting EEG power spectrum in healthy subjects. Neurosci. Lett. 419, 162-167

60 Chen, W.H. et al. (2003) Low-frequency rTMS over lateral premotor cortex induces lasting changes in regional activation and functional coupling of cortical motor areas. Clin. Neurophysiol. 114, 1628-1637

61 Vuilleumier, P. and Driver, J. (2007) Modulation of visual processing by attention and emotion: windows on causal interactions between human brain regions. Philos. Trans. R. Soc. Lond. B Biol. Sci. 362, 837855

62 Rushworth, M.F. et al. (2003) The left parietal and premotor cortices: motor attention and selection. Neuroimage 20 (Suppl. 1), S89S100

63 Schutter, D.J. et al. (2003) High frequency repetitive transcranial magnetic over the medial cerebellum induces a shift in the prefrontal electroencephalography gamma spectrum: a pilot study in humans. Neurosci. Lett. 336, 73-76

64 Pastor, M.A. et al. (2006) Modulation of steady-state auditory evoked potentials by cerebellar rTMS. Exp. Brain Res. 175, 702-709

65 Klimesch, W. et al. (2007) EEG alpha oscillations: the inhibition-timing hypothesis. Brain Res. Rev. 53, 63-88

66 Huber, R. et al. (2004) Local sleep and learning. Nature 430, 78-81

67 Massimini, M. et al. (2007) Triggering sleep slow waves by transcranial magnetic stimulation. Proc. Natl. Acad. Sci. U. S. A. 104, 8496-8501

68 Mitchell, D.J. et al. (2008) Frontal-midline theta from the perspective of hippocampal "theta". Prog. Neurobiol. 86, 156-185

69 Knyazev, G.G. (2007) Motivation, emotion, and their inhibitory control mirrored in brain oscillations. Neurosci. Biobehav. Rev. 31, 377-395

70 Kounios, J. et al. (2006) The prepared mind: neural activity prior to problem presentation predicts subsequent solution by sudden insight. Psychol. Sci. 17, 882-890

71 Melloni, L. et al. (2007) Synchronization of neural activity across cortical areas correlates with conscious perception. J. Neurosci. 27, 2858-2865

72 Miniussi, C. et al. (2008) Efficacy of repetitive transcranial magnetic stimulation/transcranial direct current stimulation in cognitive neurorehabilitation. Brain Stimulation 1, 326-336

73 Dohrmann, K. et al. (2007) Neurofeedback for treating tinnitus. Prog. Brain Res. 166, 473-485

74 Walsh, V. and Cowey, A. (2000) Transcranial magnetic stimulation and cognitive neuroscience. Nat. Rev. Neurosci. 1, 73-79

75 Lakatos, P. et al. (2008) Oscillatory entrainment as a mechanism of attentional selection. Science 320, 110-113 